\title{
Damage and Material-state Diagnostics with Predictor Functions using Data Series Prediction and Artificial Neural Networks
}

\author{
Stefan Bosse ${ }^{1, *}$ and Edgar Kalwait ${ }^{2}$ \\ 1 University of Bremen, Dept. Computer Science, Bremen, Germany, sbosse@uni-bremen.de \\ 2 University of Bremen, Faculty of Production Engineering, Bremen, Germany, ekalwait@uni-bremen.de \\ * Correspondence: sbosse@uni-bremen.de \\ + Presented at the 7th Electronic Conference on Sensors and Applications, 15 - 30 November 2020; Available \\ online: https://ecsa-7.sciforum.net/.
}

Published: 15 November 2020

\begin{abstract}
The main objective of this work is the transition of destructive material testing towards non-destructive testing methods and the investigation of predictor functions derived by Machine learning applied to destructive testing methods like tensile tests. The output provides information about the material state and damages in advance, i.e., dealing with functionals $f(x): x \rightarrow y$, where $x$ is the strain (length) and $y$ the stress (load force) variable. The scope of this work focuses on learning of predictor functions by using simple commonly used stateless forward and state-based recurrent neural networks providing two outputs separately: (1) The prediction of damage events by past recorded data with functions of the form $f\left(Y_{0}\right): Y_{0} \rightarrow x_{\mathrm{dam}}$, where $Y$ is a data point series of the load force (stress) variable $y$, and (2) The prediction of two-dimensional data point series (e.g., strainstress curves) with functions of the form $f_{\Delta}(Y): Y \rightarrow Y_{\Delta}$ and the aim to predict (extrapolate) the development of the function for a progressive difference $\Delta x$ of the strain variable. I.e., we derive new function sets $\left\{f_{1}, . ., f_{n}\right\}$ from training data to predict the material behaviour and state transitions (e.g., from elastic to plastic behaviour)..
\end{abstract}

Keywords: Material Testing, Tensile Tests, Damage Prediction, Data Series Forecasting, Recurrent Neural Networks, Material Informatics

\section{Introduction}

There is an emerging field of new materials, including, but not limited to, fibre-metal laminates, foam materials, and materials processed by additive manufacturing, highly related to a broad range of applications. Typically, material properties such as yield strength, inelastic behaviour, and damage points are determined from tensile tests.

The main disadvantage of tensile testing is the irreversible modification of the device under test (only one experiment possible!). We develop and investigate the training of approximating predictor functions by Machine Learning (ML) and simple Artificial Neural Networks (ANN) for inelastic and fatigue prediction by history recorded data. The predictor functions should be able to predict irreversible effects like inelastic (plastic) behaviour and material damage by data measured from simple tensile tests within the elastic range of the materials.

We show some preliminary results from data from tensile test experiments and outline the challenges to derive such predictor functions by using artificial neural networks and Long-short-term Memory cells (LSTM). In time-series and data-series prediction, the neural network is activated by a linearised sequence of sensor samples measured either from laboratory tensile tests or by using strain-gauge and force sensors at run-time. The predictor functions outputs an extrapolation of the 
development of the measured variables (e.g., tensile load force, stress with respect to crosssectional area).

Tensile tests (TT) are used to characterise material properties like yield criterion and the work hardening parameters to be identified, the maximal strength, elastic and non-elastic behaviour [3]. Commonly, a TT modifies the device under test (DUT) irreversible (destructive method). Only one test for each sample is possible! Non-elastic (plastic) behaviour and damage can only be detected from data measured in the past (the event happened). New hybrid and syntactic foam materials pose non-linear and unexpected behaviour hard to model on functional level.

This work investigates predictor functions for damage prediction and multi-step ahead data point series prediction derived originally from time-series prediction [5]. Artificial neural networks (ANN) are suitable models for time-series predictor functions [4] by using networks with a feedback loop from neuron outputs to input edges of neurons of the same or a previous layer, i.e., recurrent neural networks (RNN). This feedback introduces state (memory). The commonly deployed gradient-based training of simple recurrent networks is difficult due to exploding gradients. Instead, more advanced architectures with gating are utilised, e.g., Long-short term memory cells (LSTM). Typically the time domain is the series ordering variable, but any discretisable variable can be the ordering variable, e.g., the measured strain length of a tensile test. This leads to the more general data series prediction. But data series prediction can be performed with feed forward networks, too [8]. Both approaches are compared in this work,

The prediction of the plastic material behaviour in advance should be possible by sensor data from load tests acquired within the elastic material range only. The prediction of the damage point should be possible sensor data from load tests acquired within the first segment mainly consisting of the elastic range and the beginning of the first plastic segment.

The following sections introduce the methods, the model networks, the training and test techniques, followed by a discussion of analysis results from tensile tests.

\section{Aims and Methods}

\section{Data Series Processing.}

It is assumed that there are experimental methods or measuring techniques that produce a series of sensor data points related to the time, spatial, or any other physical domain. There are two different methods applied in this work to process sensor data series:

- RNN: Sequentially using state-based recurrent artificial neural network;

- FFNN: In parallel using feed forward artificial neural networks.

Both network architectures and data processing methodologies are compared in Fig. 1. The sequentially activated state-based network (commonly using Long-short term memory cells) is used for data series prediction. The parallel activated feed forward network is used to predict a damage feature. 

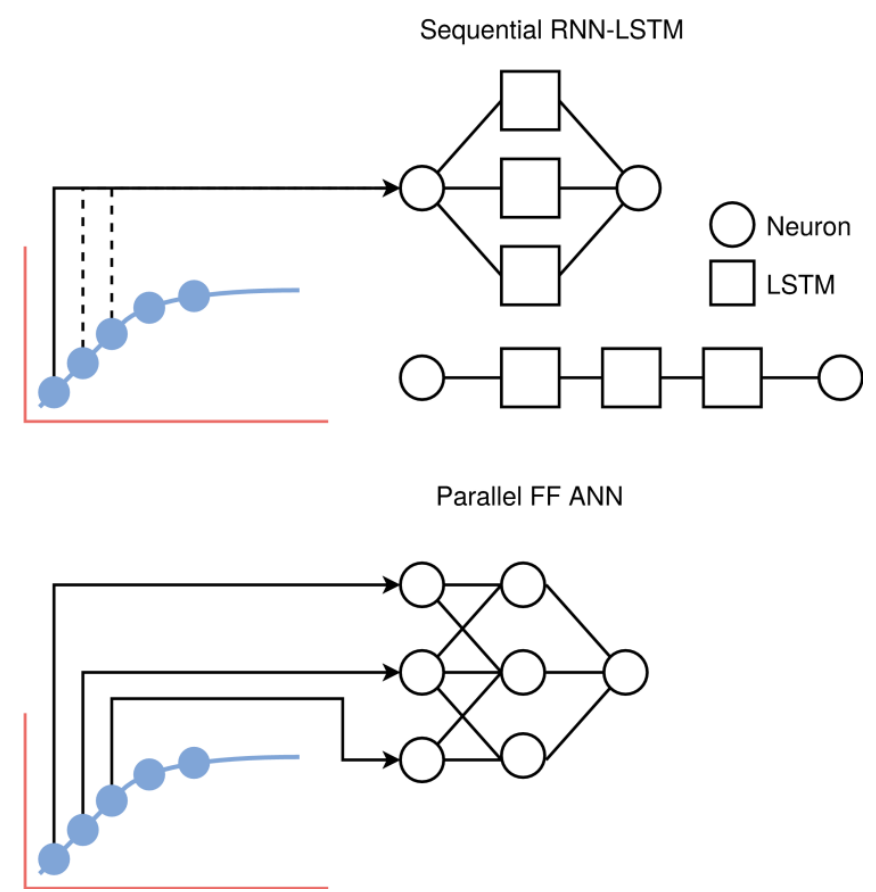

Fig. 1. Sequential versa parallel processing of data points of measured sensor data series

\section{Damage prediction.}

The first approach discussed in this work utilizes data points from tensile tests within the first segment (basically the elastic and the beginning of the first plastic range). From this first segment a small number $(<20)$ of feature points is extracted and used to predict the strain length $x_{\text {dam }}$ where the specimen will break (fatal damage). This point is defined by a rapid decrease of the load force towards zero.

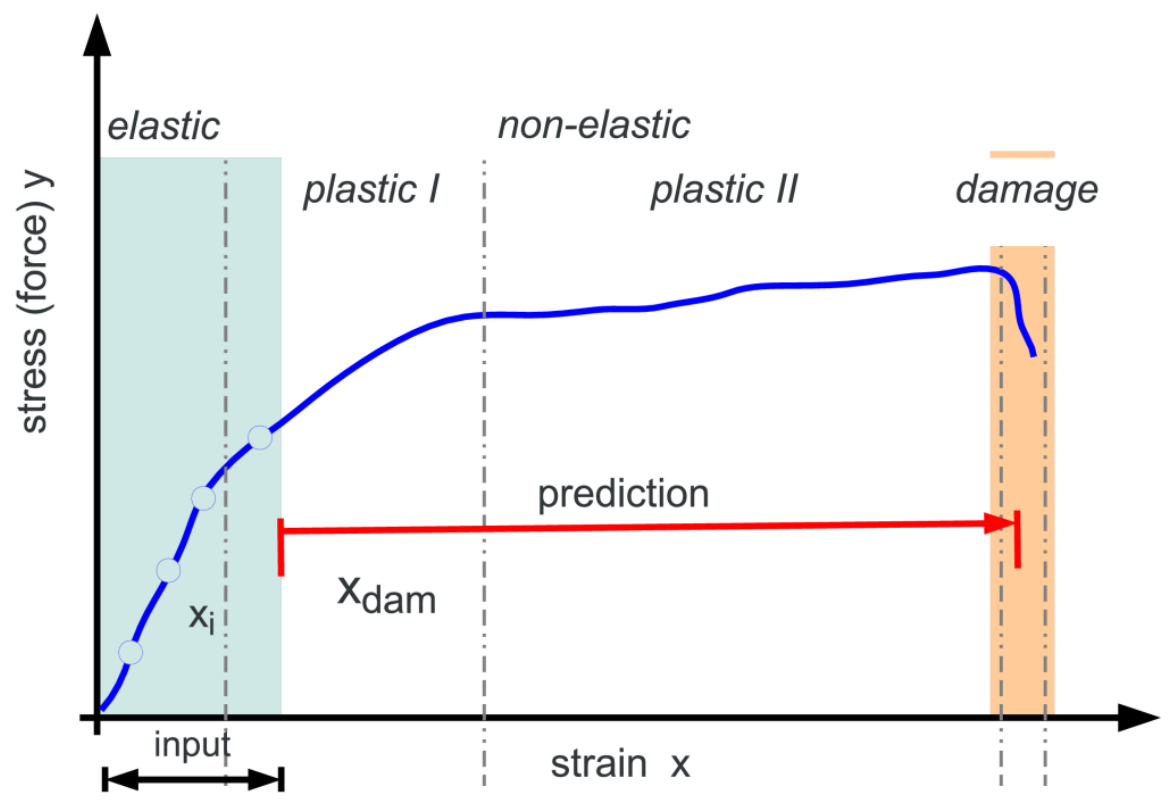

Fig. 2. Damage point prediction (strain length $x$ ) from measured data of the first segments of the strain-stress diagram from tensile tests

\section{Material behaviour prediction.}

The second approach utilizes data point series prediction to recognize material state transitions like the transition from elastic to plastic material behaviour in advance. This requires an accurate prediction of the strain-stress (or strain length and load force) functions of a specific specimen. The 
entire strain-stress curve of a specimen is derived from data series of $\langle x, y\rangle$ tuples where $x$ is the strain length and $y$ the load force variable.

Learning a set of predictor model functions $\left\{f_{1}, \ldots, f_{n}\right\}$ with $f_{\delta}(Y): Y \rightarrow Y_{\delta}$ with $Y_{\delta} \supset Y$ from strainstress curves requires state-based predictor functions. There is $Y=\left\{y_{1}, y_{2}, . . y_{i}\right\}$. One common model is a recurrent artificial neural network with Long-short term memory cells (LSTM), already successfully deployed in data point series prediction for damage event diagnostics [1] [4].

$Y$ is a series of equally spaced $y$-values $\left\{y_{1}, . ., y_{n}\right\}$ with respect to the $x$ variable, and $Y_{\delta}$ is a shifted

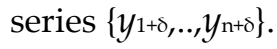

The trained models can be used to predict the future development based on past data of:

1. The stress (load forces) acting on the sample related to the observed strain;

2. The strain (length) related to observed stress or measuresd load forces (inverse problem).

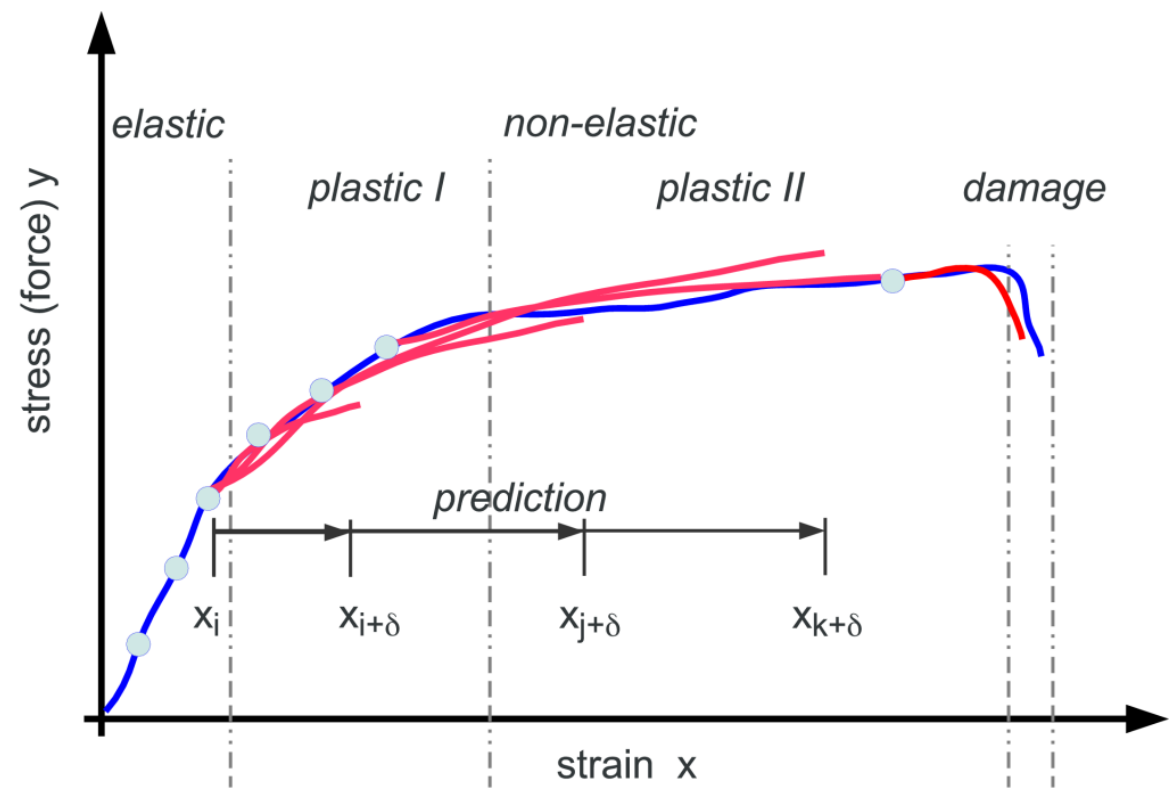

Fig. 3. A typical measured strain-stress curve from a tensile test (blue line) and forward predictions

(red line segments) $x_{\mathrm{i}+\delta}$

Each predictor function $F$ of the set \&Fopf; is able to predict the target variable at $i+\delta$ steps ahead assuming a descritization using the past target variable values (measured), i.e., performing an extrapolation of the function for future function values:

$$
\forall \delta \in\{1,2, . ., m\}: y(i+\delta)=F_{\delta}\left(y_{0}, y_{1}, . ., y_{i}\right)
$$

Input and output neurons of continuous predictor functions uses typically a sigmoid activation function to provide a nearby linear transmission in the desired output range.

\section{Experimental Data}

The following experiments were carried out with data from tensile test made with aluminium sheets (approx. $5 \times 12 \times 1 \mathrm{~mm}$ size). There are three series of specimens (Fraunhofer IFAM, Lehmhus et al.). A reference series $R$, and two thermally treated specimens with the series $F$ and $T$. The samples of the series $\mathrm{F}$ and series $\mathrm{T}$ are made of heat treated 7075 aluminium sheets with dimensions of approx. $5 \times 12 \times 1 \mathrm{~mm}$. In the case of series $\mathrm{F}$, the sheets were quenched directly after pouring in $20^{\circ} \mathrm{C}$ warm water. After quenching and an aging process at room temperature, the series- $\mathrm{T}$ was again thermally temperature-controlled at $170{ }^{\circ} \mathrm{C}$ for 8 days. The aluminium sheets of the reference series 
have not undergone any thermal treatment. The plates of the individual series were clamped at different angles during the tensile tests in order to achieve a variance.

One main issues with data sets from tensile test is the low degree of variance. Typically, the (labelled) data set is split in training and test sub-sets.

\section{Data Normalization and Augmentation.}

Data series prediction uses commonly series of data tuples $<x, y>$, e.g., with $x=t$ (time variable). The prediction is applied only to a sequence of $y$ values. In this work, the ordering variable $x$ is a sensor variable from the tensile test (strain length), and $y$ is the target variable to be predicted (load force or stress). The original sensor data series must be linearised with respect to the $x$ variable, i.e., the final data series contains only equally spaced force values with respect to the measured strain length, i.e., a constant $\Delta x(y i, y i+1)=\delta$

The synthetic data augmentation is a possibility to generate additional data sets from a few measured data sets, typically the situation in tensile test experiments. The first consideration is simply to stretch or compress one or more existing curves linearly on the force axis. This approach leads to a too small variation of the synthetic data. Monte Carlo simulation can add randomness to measured data and can extend the training data base significantly. This method is an established method to avoid the trend to specialisation of predictor functions during training.

The second approach uses the consideration of a weighted averaging of two or more curves to generate new data series. This can vary due to the different weighting of the respective curves. Thus, it is possible to generate infinitely many new synthetic curves.[7] to do this, a "Dynamic Time Warping" (DTW) path is generated from two random curves, which is then used to average the points of the two curves assigned to each other. The DTW path is constructed as an Array of tuples, where the first element of the tuple is the point of the first curve and the second element is the associated point from the second curve [6].

\section{Predictor Functions and Models}

Predictor functions can be modelled using mathematical functions or using ANN to approximate these functions. A modified Neataptic ML framework was used to implement FFNN and LSTM-RNN and to perform training and prediction [9].

\section{Feed Forward Networks.}

For the damage point prediction a parallel activated ANN is used approximating the hypothesis function $f\left(y_{0}, . . y_{n}\right): \mathbf{y} \rightarrow x_{\text {dam }}$ consists of $n$ (e.g., $n=18$ ) input neurons (each connected with one of the data points from the load force variable $y$ ), one ore more inner layers, and one output neuron. Good prediction results were achieved with the neuron-layer configuration [ $n: 7: 3: 1]$. I.e., the first $n$ down sampled data points $\left\{y_{1}, y_{2}, . ., y_{n}\right\}$ are used to predict the distance $x_{\text {dam }}$ to a data point related with the damage (break) event.

Alternatively a sequentially activated recurrent ANN with memory cells is used for comparison. The architecture is discussed in the next section.

\section{Recurrent Networks.}

Another aim of this work is to derive a predictor function that is able to predict a curve trace in advance for a given number of steps (delta of the input variable relative to the output variable). Extrapolation requires either a fitted function model, i.e., solving a regression problem, or state-based model that remembers and recognizes typical curve trace segments to give an approximation of the extrapolation of these segments. Regression by function fitting results typically in specialized model. More generalized models can be approximated in a more straight forward way by state-based ANN well known for time-series prediction. 
The ANN consists of an input layer (only one neuron), a hidden LSTM layer (or more), and one output layer (only one neuron). The input variable of the network is a sequence of $y$ values, i.e., measured forces. The output variable of the network is the predicted $y$ value for a future $x$ point.

The hidden layer consists of LSTM cells which are connected with the previous and next layer (optional connection between MC in same layer). The sequence samples must be normalized (equally spaced) with respect to the also measured strain length $x$ !

The memory cell of the LSTM is connected to several gates. The gates control connection weights dynamically (like a valve). There are different optional LSTM network configurations effecting the interconnection of LSTM cells with each other.

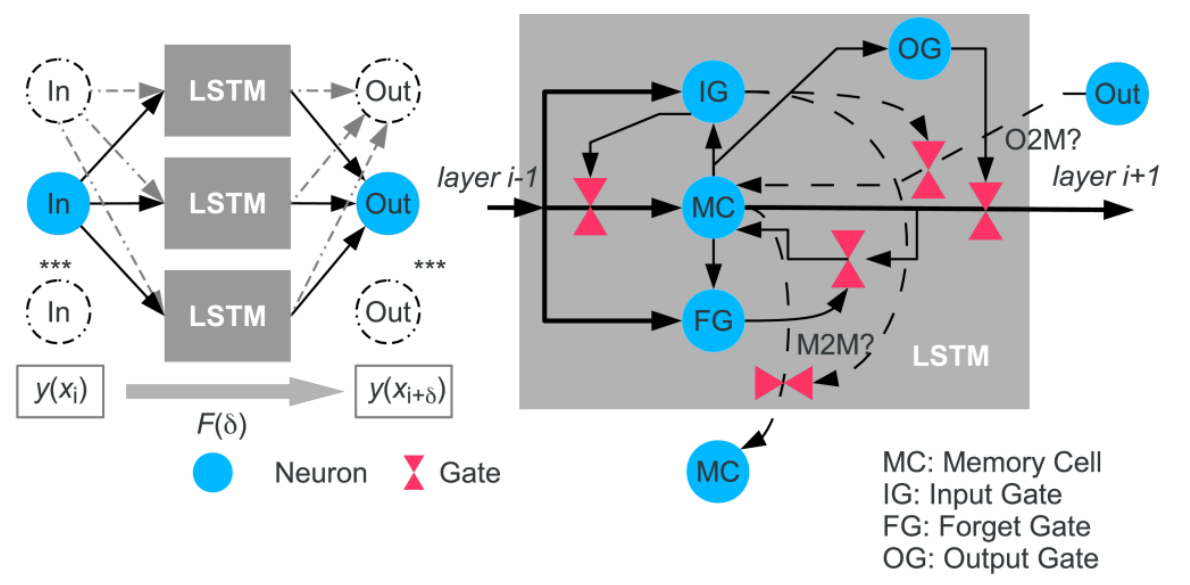

Fig. 4. The sequentially activated LSTM-RNN implementing the predictor functions $f_{\Delta}$ to predict material behaviour and state transitions

Different network configurations were tested. Finally, a LSTM cell chain with a horizontally linear configuration [1:1:1:1:1:1:1:1:1:1:1:1] with one input, one output neuron, and a chain of $n=10$ connected LSTM cells were chosen for the generalised $y_{\diamond}$ variable prediction (supporting a broad range of material variations). Another approach for a specialised predictor function (supporting only one specimen class) used a vertically expanded configuration, e.g., [1:3:4:1].

\section{Training and Test}

\section{Experimental Data.}

The original raw experimental data from the tensile tests were pre-processed by $x$-variable normalisation, augmentation by Monte Carlo simulation and dynamic eime warping techniques, and finally $y$-variable normalisation to the value range $[0,1]$.

\section{Feed Forward Network.}

The FFNN was trained with a sequence of randomly selected of training examples by using a classical gradient based error back propagation algorithm. The test was performed with respect to the target output variable ${ }^{*} \mathrm{x}^{*} \sim$ dam $\sim$ compared with the value computed numerical from the full tensile test curve.

\section{Recurrent Network.}

The RNN were trained with a sequence of randomly selected training examples. In the case of the damage point target variable, all data points of a sequence (down sampled to only a few points, $n<100)$ activated the RNN sequentially. After activation, an error back propagation step was performed (comparing actual and target output value). 
The data point series prediction $y(x+i)$ uses a single data point activation with immediate error back propagation. The test was performed with all data sets by comparing the predicted with the real output values.

\section{Results}

The trained predictor functions are evaluated with respect to the two target variables damage point and load force series prediction.

\section{Damage prediction.}

\section{Forward Parallel Network.}

The in parallel activated FFNN shows a high accuracy of the damage point prediction with low variance. The training was performed (1) With a random 1:1 split of training and test data from the entire data set consisting of 3 different series, and (2) with all experiments. The test was performed with all experiments. The average accuracy is in both cases about $9 \%$ (max.: $30 \%, \sigma=7 \%$ ). Results are shown in Fi.g 5.

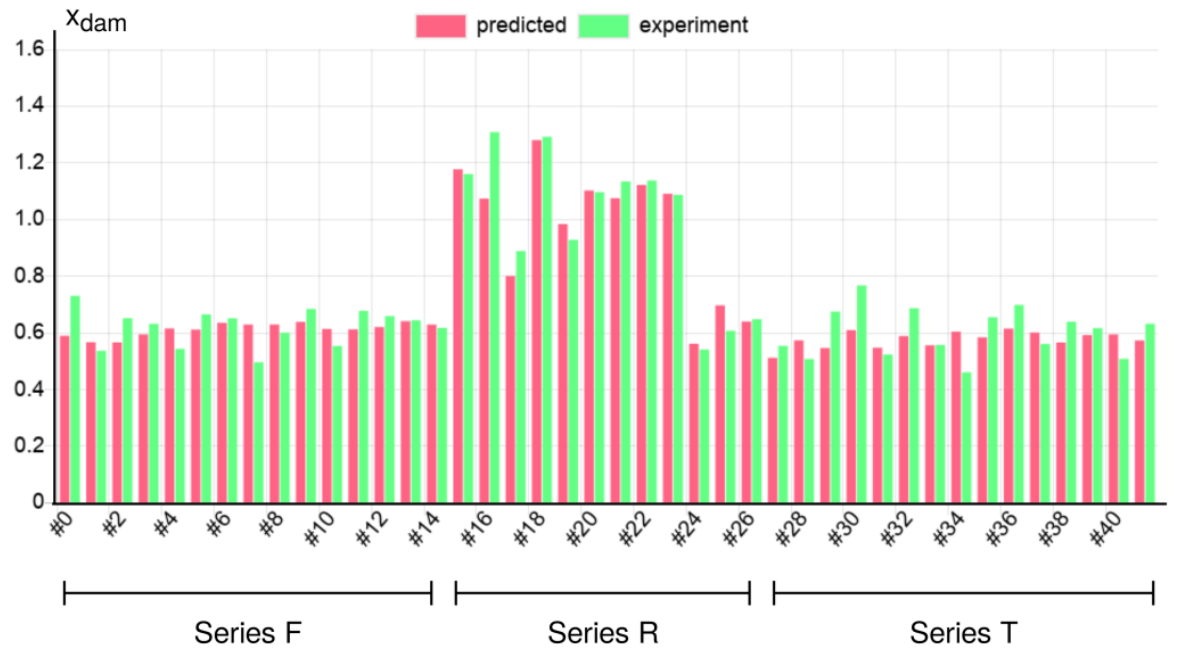

Fig. 5. Prediction results for the damage length point $x_{\text {dam }}$ of different specimens and series using an in parallel activated feed forward network

\section{Recurrent Sequential Network.}

the same test and the same training data was made with a sequentially activated LSTM-RNN, too. This method shows low average accuracy about $15 \%$ not suitable to predict the damage point. Results are shown in Fi.g 6.

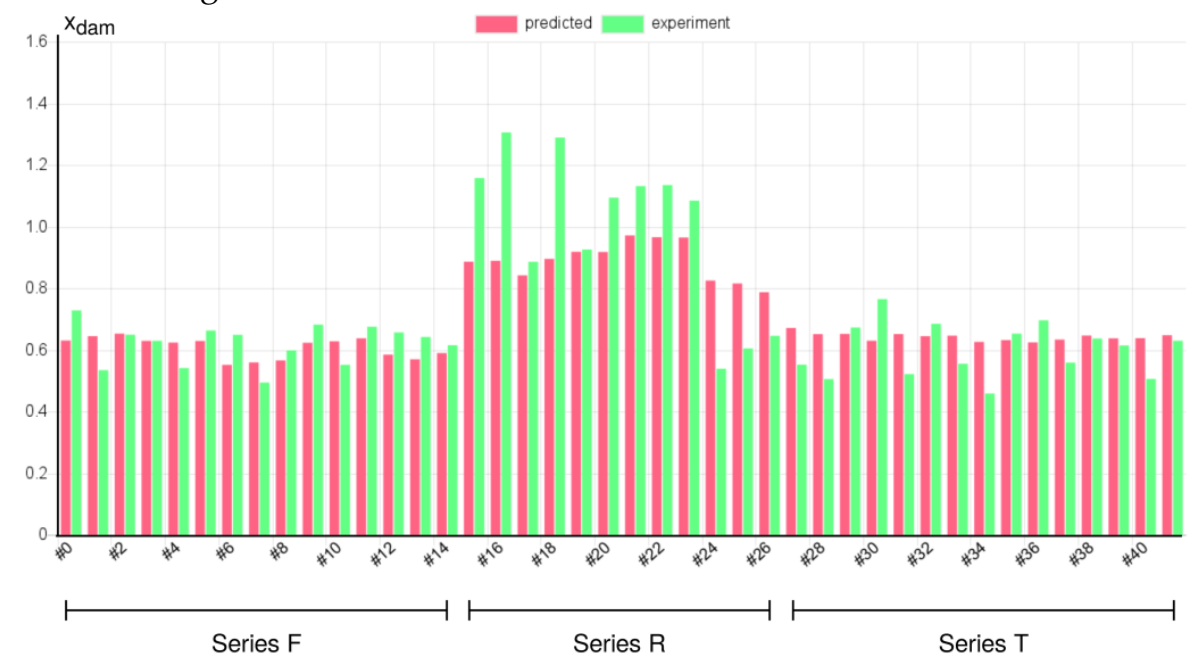


Fig. 6. Prediction results for the damage length point $x_{\text {dam }}$ of different specimens and series using a sequentially activated LSTM-RNN network

Material behaviour prediction.

TT from 42 experiments of metal sheets with different thermal preparation were used as input data for training and testing of the LSTM-RNN predictor function.

Example plots of measured (red line) and predicted $x-y$ curves (green line) for different specimens and series is shown in Fig 7. The predictor function was trained with data from all series and using all experiments of each series.

The maximal prediction error of the predictor function for $\delta=25$ (about $3 \%$, equal to $25 \mu \mathrm{m}$ ) sample points (full scale of measurement ranges between 700 and 1400 points) is below $10 \%$, with an average of less than $5 \%$. The prediction deviates more strongly in curve segments with a high gradient (with spikes, too).

Higher number of hidden LSTM layers can improve prediction accuracy. Although the error between the original and the predicted strain-force curves is lower than $10 \%$, the predicted curves tend to be delayed (positive shift on $x$-axis) and it is difficult to predict the beginning of the inelastic material behaviour in the elastic segment. This a result from the short elastic segment compared with the long inelastic segment, too.
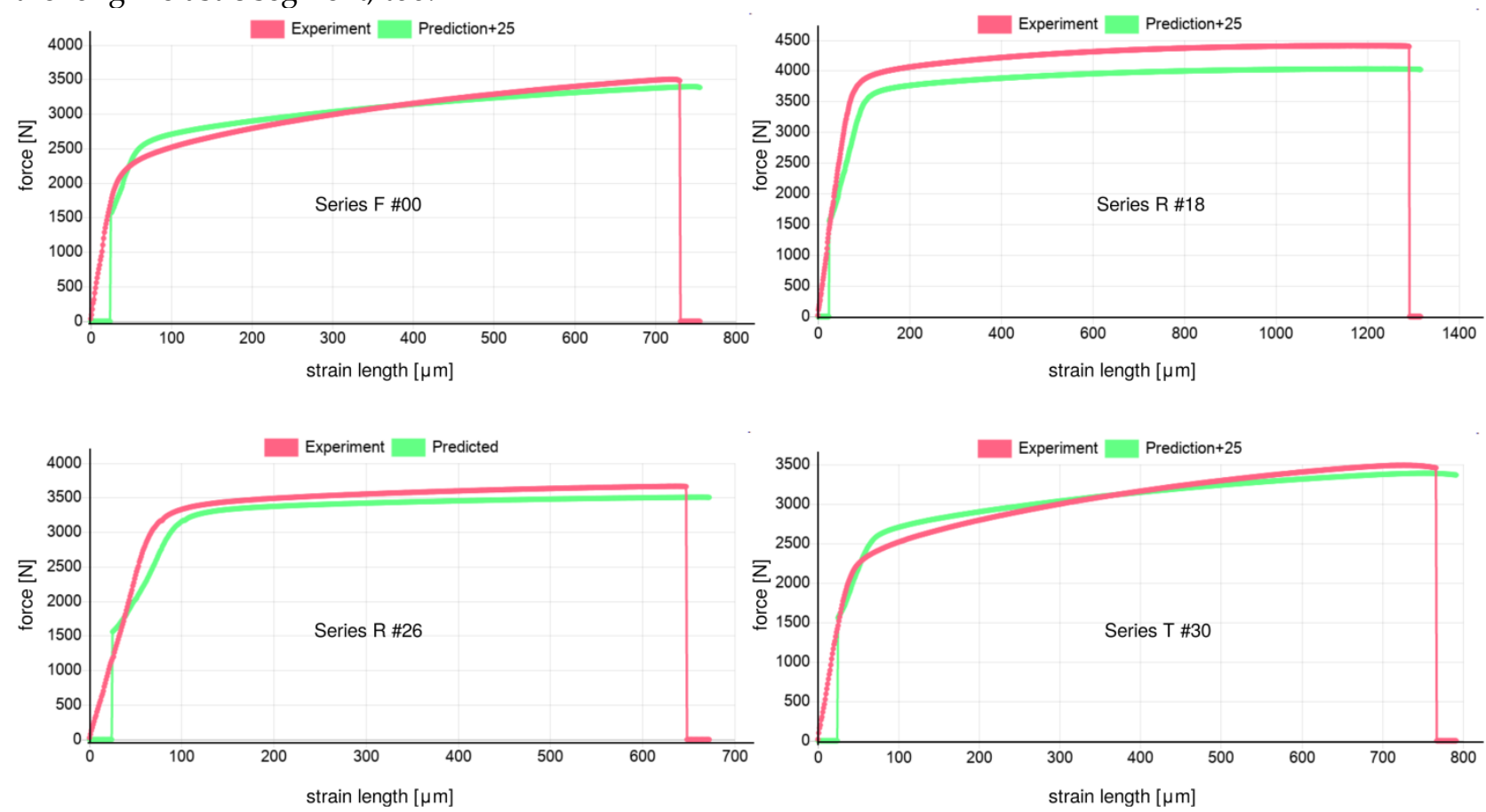

Fig. 7. Results from the material strain-stress behaviour prediction of different specimens and series using a sequentially activated chain LSTM-RNN network with $\delta=25 \mu \mathrm{m}$ (equal to 25 data points)

In Fig. 8 samples of prediction results are shown with training and prediction using only one series $\mathrm{R}$ with different model network configurations and without data augmentation. In contrast to the models trained by all specimens and over all series (previous results), this limitation to a specific series enables the detection of the beginning of the plastic segment with data taken from the elastic range. The predicted curves in Fig. 8 do not pose the prediction lag from the previous curve predictions. But a proper design of the network architecture is eminent for accurate predictions. 

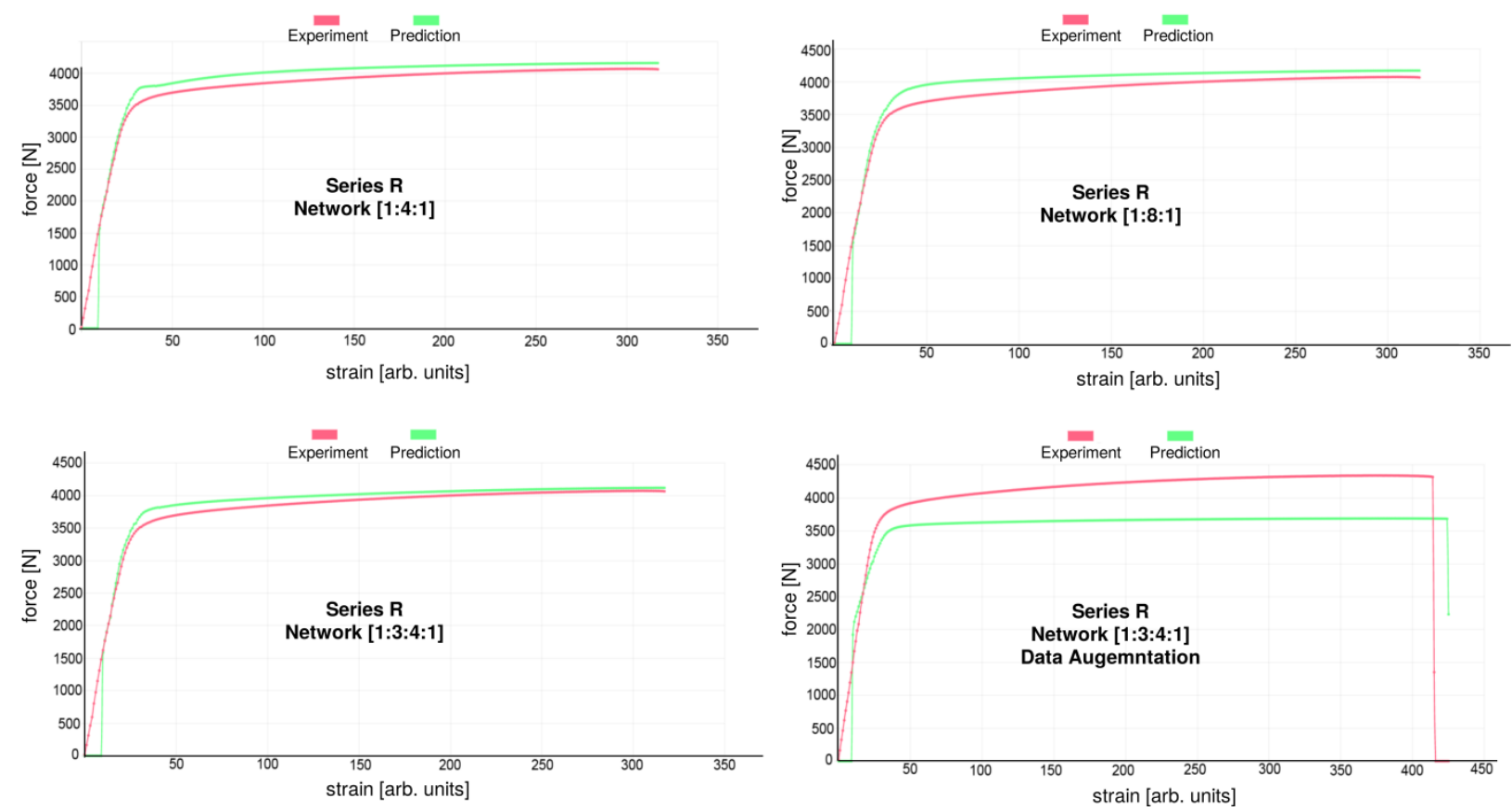

Fig. 8. Results from the material strain-stress behaviour prediction of one specimen from series $\mathrm{R}$ using a sequentially LSTM-RNN network with $\delta=10$ data points (approx. $25 \mu \mathrm{m}$ ) and different model netwrok configurations [6]

\section{Conclusion}

Simple forward and recurrent neural networks are evaluated for learning functions for predicting the future development of material behaviour under load conditions, i.e., prediction of damage points (by estimating the strain length where the damage will occur) and material state transitions like elastic to inelastic material behaviour based on past measured force and strain length value sequences (mostly in the elastic material range). A classical in parallel activated feed forward network was able to predict the damage point by the first segment of data points of the strain-stress curve with a high accuracy. The network was activated by the data points in parallel. Despite the common understanding that RNN-LSTM are suitable candidate models for time and data point series prediction, they could not be trained successfully to predict damage points and material state transitions in a general way (covering different material and specimen properties by one model). Here the data points activate the network sequentially. Specialized models limited to specific specimen, material and treatment parameters were in principle able to predict the plastic material behaviour with data points from the elastic segment.

\section{References}

1. S. Bosse, Learning Damage Event Discriminator Functions with Distributed Multi-instance RNN/LSTM Machine Learning - Mastering the Challenge, Proc. of the 5th International Conference on System-Integrated Intelligence Conference, Bremen, Germany, 2020

2. S. Bosse, D. Lehmhus, Robust detection of hidden material damages using low-cost external sensors and Machine Learning, 6th International Electronic Conference on Sensors and Applications (ECSA), 15-30 Nov. 2019, MDPI

3. B.M. Chaparro, S. Thuillier, L.F. Menezes, P.Y. Manach, J.V. Fernandes, Material parameters identification: Gradient-based, genetic and hybrid optimization algorithms, Computational Materials Science, Volume 44, Issue 2, 2008

4. Y. Hua, Z. Zhao, R. Li, X. Chen, Z. Liu and H. Zhang, Deep Learning with Long Short-Term Memory for Time Series Prediction, in IEEE Communications Magazine, vol. 57, no. 6, pp. 114-119, June 2019, doi: 10.1109/MCOM.2019.1800155. 
5. H. Cheng, P.-N. Tan, J. Gao, and J. Scripps, Multistep-Ahead Time Series Prediction, in Pacific-Asia Conference on Knowledge Discovery and Data Mining, PAKDD 2006: Advances in Knowledge Discovery and Data Mining, 2006.

6. E. Kalwait, Entwicklung einer Prädiktorfunktion zur Vorhersage einer Zielvariablen aus Sensordaten im Kontext der Materialprüfung, 2020, Bachelor thesis, University of Bremen

7. G. Forestier, F. Petitjean, H. A. Dau, G. I. Webb and E. Keogh, Generating Synthetic Time Series to Augment Sparse Datasets, 2017 IEEE International Conference on Data Mining (ICDM), New Orleans, LA, 2017, pp. 865-870, doi: 10.1109/ICDM.2017.106.

8. Z. Tang and P. A. Fishwick, Feed-forward Neural Nets as Models for Time Series Forecasting, TR91-008 Computer and Information Sciences, University of Florida.

9. T. Wagenaar, online, https://wagenaartje.github.io/neataptic, accessed 1.1.2020

(C) 2020 by the authors. Submitted for possible open access publication under the terms and conditions of the Creative Commons Attribution (CC BY) license (http://creativecommons.org/licenses/by/4.0/). 